\title{
Stabilization of $\mathrm{MgAl}_{2} \mathrm{O}_{4}$ spinel surfaces via doping
}

\author{
Md. M. Hasan ${ }^{a}$, Pratik P. Dholabhai ${ }^{b}$, Ricardo H. R. Castroa, Blas P. Uberuagab \\ a. Department of Chemical Engineering and Materials Science, University of California, Davis, \\ CA 95616, USA. \\ b. Materials Science and Technology Division, Los Alamos National Laboratory, Los Alamos, \\ NM 87545, USA.
}

\begin{abstract}
Surface structure of complex oxides plays a vital role in processes such as sintering, thin film growth, and catalysis, as well as being a critical factor determining the stability of nanoparticles. Here, we report atomistic calculations of the low-index stoichiometric magnesium aluminate spinel $\left(\mathrm{MgAl}_{2} \mathrm{O}_{4}\right)$ surfaces, each with two different chemical terminations. High temperature annealing was used to explore the potential energy landscape and provide more stable surface structures. We find that the lowest energy surface is $\{100\}$ while the highest energy surface is $\{111\}$. The surfaces were subsequently doped with three trivalent dopants $\left(\mathrm{Y}^{3+}, \mathrm{Gd}^{3+}, \mathrm{La}^{3+}\right)$ and one tetravalent dopant $\left(\mathrm{Zr}^{4+}\right)$ and both the surface segregation energies of the dopants and surface energies of the doped surface were determined. All of the dopants reduce the surface energy of spinel, though this reduction in energy depends on both the size and valence of the dopant. Dopants with larger ionic radius tend to segregate to the surface more strongly and reduce the surface energy to a greater extent. Furthermore, the ionic valence of the dopants seems to have a stronger influence on the segregation than does ionic size. For both undoped and doped spinel, the predicted crystal shape is dominated by $\{100\}$ surfaces, but the relative fraction of the various surfaces changes with doping due to the unequal changes in energy, which has implications on equilibrium nanoparticle shapes and therefore on applications sensitive to surface properties.
\end{abstract}

\section{INTRODUCTION}

Understanding the surface structure of complex oxides is important not only for processes that occur on surfaces, such as catalysis and film growth, but also for composite configurations in which the oxide surfaces are brought into contact with other metals or oxides, such as metal/oxide and oxide/oxide thin film structures. Metal/oxide and oxide/oxide interfaces play a key role in a vast array of technologically important applications such as composites, protective coatings, thin film technology, electronic packaging, gas sensors, oxide supported transition metal catalysts, multiferroics, nanostructured ferritic alloys, etc [1-5]. For the majority of these diverse applications, fundamental knowledge of the structure and properties of the surfaces and interfaces is critical for identifying and designing materials with superior functionalities. The properties of a given surface are often summarized in one quantity, the surface energy. For oxide ceramics, this property is critical as it controls many chemical and physical processes and 
properties. Knowledge of this property can aid in predicting and optimizing processing parameters and properties [6]. Moreover, the nano-scale behavior of materials is highly dependent on surface energy, as at the nano-scale materials often have very high surface-tovolume ratios and are dominated by surface effects.

The surface structure and consequently the surface energy of materials can be controlled using dopants that segregate to, and thus modify, the surface. The reduction in surface energy through dopant segregation can be quantified using the well-known Gibbs adsorption isotherm [7]:

$$
d \gamma=-\Gamma_{A} d \mu_{A}
$$

where $\gamma$ is the surface energy, $\mu_{A}$ is the chemical potential of dopant $A$ dissolved in a material with a constant number of atoms, and $\Gamma_{A}$ is the excess amount of dopant $A$ at the surface compared to the bulk.

Both theoretical and experimental works have been reported showing solute segregation to grain boundaries and consequent reduction of grain boundary energy [8-10]. Using this approach, thermodynamic stabilization of grain boundaries has been achieved. In more recent work, surface segregation of dopants and corresponding surface energy reduction was experimentally verified in the $\mathrm{CeO}_{2}$ and $\mathrm{SnO}_{2}$ systems $[11,12]$.

$\mathrm{MgAl}_{2} \mathrm{O}_{4}$ spinel, an oxide ceramic material with the chemical formula $A^{2+} B_{2}^{3+} O_{4}^{2-}$ is a technologically vital material because of its high thermal stability, chemical inertness, and mechanical strength, in particular as compared to the isotructural catalyst support material $\gamma$ $\mathrm{Al}_{2} \mathrm{O}_{3} . \mathrm{MgAl}_{2} \mathrm{O}_{4}$ has been used for Pt catalyst support, exhibiting remarkable results in stabilizing Pt nanoparticles [13, 14]. Recently, the role of the spinel surface structure in stabilizing Pt nanoparticles was demonstrated through experiments as well as density functional theory (DFT) calculations [15]. It was found that small Pt particles (1-3 nm) deposited on $\{111\}$ spinel surfaces can survive severe aging, maintaining high Pt dispersions, while those on $\{100\}$ surfaces are sintered. The type of surface termination and epitaxial growth of Pt particles on $\{111\}$ helped in the stability of Pt, which led to the conclusion that enhancing the quantity of spinel $\{111\}$ facets in a magnesium aluminate support can help in designing platinum group catalysts.

Several works have been reported on the surface structures of spinels [16-20]. In the case of $\mathrm{MgAl}_{2} \mathrm{O}_{4}$ spinel, atomistic and DFT calculations were used to understand the structure and energy of low-indexed surfaces [16-18, 21]. Regarding dopant segregation, some researchers reported grain boundary segregation of several rare earth dopants in spinel [22, 23]. Using atomic resolution imaging coupled with single atom analytical detection capabilities, they reported a single layer of rare earth atoms at the grain boundary with a segregated region of $<0.4$ $\mathrm{nm}$. However, none of these studies examined how dopant segregation depends on surface structure or the nature of the dopant. Further, how these dopants stabilize the complex surfaces of spinels is not clear. 
In this work, using atomistic calculations, we aim to understand the relative segregation tendency of several dopants at low-index surfaces of stoichiometric $\mathrm{MgAl}_{2} \mathrm{O}_{4}$ spinel. We begin by constructing models of the three low-index surfaces, accounting for the possible different terminations, similar to the approach taken in Ref. [16]. We then determine how dopants segregate to these surfaces in order to predict how those dopants modify the surface energetics. Finally, we use these results to construct Wulff plots [24], which demonstrate the influence of dopant segregation on the surface morphology of spinel particles.

\section{METHODS}

Atomistic calculations with three-dimensional periodic boundary conditions (PBC) were performed within the framework of LAMMPS [25]. The calculations are based on energy minimization using a classical Born-like description of an ionic lattice. The ionic interactions have two components: long-range Coulombic pair terms, which assume a full ionic charge, and short-range interactions accounting for the effects of electron cloud overlap and van der Waals interactions. Two-body short-range interactions were described using parameterized Buckingham [26] pair potentials while long-range Coulombic (electrostatic) forces were summed using Ewald's method [27]. Buckingham interatomic potentials parameters were taken from the work of Busker et al. [28].

Previous studies on spinel surfaces were done on low-index planes of the cubic structure, which in general represent lowest energy structures $[16,17]$. Consequently, we have examined three low-index planes, the $\{100\},\{110\}$, and $\{111\}$ surfaces of spinel, to predict the surface energy and compute segregation profiles for one aliovalent $\left(\mathrm{Zr}^{4+}\right)$ and three isovalent $\left(\mathrm{Y}^{3+}, \mathrm{Gd}^{3+}\right.$, and $\mathrm{La}^{3+}$ ) dopants to these surfaces. As a first step, we constructed atomic models of spinel surfaces. All low index surfaces of spinel are of type III in Tasker's notation [29], indicating that all exhibit a surface dipole in their ideal termination. Nonetheless, the basic requirement for the calculation is that the simulation cell (which is constructed in a typical slab geometry) does not have a dipole perpendicular to the surface, since such a dipole when repeated into the crystal would result in the divergence of the surface energy [1, 30, 31]. To remove the surface dipole moment, by ensuring that both surfaces have equal charge distribution, surface vacancies were created wherein half of the surface atoms of each species were shifted to the opposite surface.

For each low-index surface, several terminations are possible, based on ideally cleaving the crystal at different atomic planes [17]. For each surface, we considered two terminations, schematically represented in Figure 1, where the planes where the cleaving was done are labeled as ' $a$ ' and 'b', after the notation used by Davies el al. [16]. That is, for an 'a' surface, the crystal was cleaved such that the 'a' plane was the surface plane. In the $\{100\} \mathrm{b}$ termination, half of the $\mathrm{Mg}$ ions from the top layer were shifted to the bottom layer (opposite surface) while, for $\{100\} \mathrm{a}$, half of the $\mathrm{Al}$ and $\mathrm{O}$ ions from the top layer were shifted to the bottom layer. For the $\{110\}$ surface, in the $\{110\} \mathrm{b}$ termination half of the atoms ( $\mathrm{Al}, \mathrm{Mg}$, and $\mathrm{O})$ from the mixed layer of the top surface were shifted to the bottom surface and in the $\{110\}$ a termination, this was done on 
the Al-O layer at the surface. For the $\{111\}$ surface, for both $\{111\} \mathrm{b}$ and $\{111\}$ a terminations, $\mathrm{Al}$ cations were removed but from two different types of $\mathrm{Al}$ surface layers.

As mentioned, for each of the six terminations described above, we removed the surface dipole formed in the ideal surface by moving half of the ions from the top surface to the bottom surface. However, this is not a unique process as there are numerous possible arrangements of the resulting surface vacancies and the energetics of the surface certainly depend on that arrangement [32]. For each termination, we took the vacant site arrangement producing the lowest surface energy found in the work of Davies et al. [16]. For instance, the top view (along the [100] axis) of the ideally terminated $\{100\}$ b surface cleaved along the $\mathrm{Mg}$ layer is shown in Figure 2(a). Figure 2(b) shows the as-constructed dipole free surface termination where half of the $\mathrm{Mg}$ ions are removed from this layer and shifted to the opposite surface in the slab model. (In Fig. 2, we highlight the $\{100\}$ b surface as we find it to be lowest in energy.)

Employing this procedure, a stoichiometric slab (e.g. $1.6 \times 1.6 \times 6.4 \mathrm{~nm}^{3}$ for $\left.\{100\}\right)$ of $\mathrm{MgAl}_{2} \mathrm{O}_{4}$ was built. To mimic the surface, a vacuum of $5 \mathrm{~nm}$ was added along the Z-axis (normal to the surface). Thus, in constructing our models, three constraints were imposed. First, stoichiometry was maintained; no atoms were added or removed from the original structure. Second, the surfaces were modified, as described, to remove any surface dipole. Finally, the two resulting surfaces in the slab structure had the same initial atomic structure, prior to any further minimization procedure. That is, as constructed, both surfaces in the model had the same structure. We note that these constraints mean that only Al-terminated interfaces are possible for the $\{111\}$ orientation as, for any other termination, the two surfaces would not be equivalent while maintaining stoichiometry.

The optimized structure (Figure 2(c) for the case of $\{100\}$ b) of each stoichiometric undoped spinel surface was obtained via energy minimization in which all three dimensions of the simulation cell as well as the forces on all atoms were allowed to relax. In addition, the surfaces were annealed at $2000 \mathrm{~K}$ for $500 \mathrm{ps}$, followed by slow cooling, to further equilibrate the structure and to allow the surface vacancies to rearrange. As we will describe below, the high temperature annealing also permits some ions to move between layers, as shown in Figure 2(d) in which significant movement of oxygen from the subsurface layers to the top-most surface layer is observed. This extra rearranging of ions typically further stabilized the structure, resulting in a surface with lower energy. Thus, surfaces that were annealed and then relaxed (energy minimization) were used for all subsequent calculations.

Once stable surface structures were obtained, segregation profiles of three different trivalent dopants $\left(\mathrm{Y}^{3+}, \mathrm{Gd}^{3+}\right.$, and $\left.\mathrm{La}^{3+}\right)$ and one tetravalent dopant $\left(\mathrm{Zr}^{4+}\right)$ were calculated at each of the six low-index surfaces. In doing so, one $\mathrm{Al}^{3+}$ ion was replaced by one dopant ion, one-by-one, and energy minimization was performed wherein the atomic positions were allowed to fully relax (though the cell dimensions were held constant) prior to computing the energy of the system. Thus, every Al atom starting from the bottom surface to the top surface of the cell was replaced 
by a dopant, and the corresponding energy of the minimized system was computed. The energy difference between the structure with a dopant in the bulk interior of the system and that with a dopant at the surface is defined as the surface segregation energy of the dopant per dopant atom, $E_{\text {seg. }}$. We have used the convention that a positive surface segregation energy indicates that the energy of the dopant is lower at the surface than in the bulk.

The surface energies of the updoped spinel were calculated from the energy difference between a slab structure with two surfaces $\left(\mathrm{E}_{\text {surf }}\right)$ and a bulk structure without any surface having the same number of atoms ( $\mathrm{E}_{\text {bulk }}$ ) by using the following relationship:

$$
\gamma=\frac{1}{2 A}\left(E_{\text {surf }}-E_{\text {bulk }}\right)
$$

where $A$ is the surface area. The factor of 2 accounts for the fact that we have 2 surfaces in our model.

The surface energies of the doped surfaces were calculated from the surface energies of the undoped surfaces and the surface segregation energy of the dopant as follows (per Ref. [33]):

$$
\gamma_{\text {doped }}(n)=\gamma-(n / s) E_{\text {seg }}
$$

where $\gamma_{\text {doped }}(n)$ is the surface energy of the doped surface, $\gamma$ is the surface energy of the undoped surface, $E_{s e g}$ is the segregation energy per dopant atom, and $n$ is the number of dopants present in a surface area of $s$. In this work, we arbitrarily assume one dopant atom per $1 \mathrm{~nm}^{2}$ surface area for all of the calculations.

Finally, after obtaining the surface energy of each surface both with and without dopants, the equilibrium crystal morphology was constructed using Wulff's method [24]. In this approach, the crystal shape that minimizes the overall surface energy is the shape such that $h_{i}$, the normal vector to the face from a point within the crystal, would be proportional to the surface energy of that face, $\gamma_{i}$,

$$
h_{i}=\lambda \gamma_{i}
$$

Here, $\lambda$ is a constant that depends on the size of the crystal. Assuming an arbitrary crystal size much greater than the spinel unit cell size, the normal vector lengths from each of the three faces ( $\{100\},\{110\}$, and $\{111\})$ were calculated using their respective surface energies and then the crystal shape was determined. Crystal shapes for the minimized surfaces, annealed-plusminimized surfaces, and doped surfaces were predicted using this method. For each low-index surface, the energy of the lower-energy termination was used.

\section{RESULTS AND DISCUSSION}

\section{Relaxation and Annealing}


Figure 2(b) and 2(c) show the as-constructed dipole-free termination and the relaxed (minimized only) structures for the $\{100\} \mathrm{b}$ surface, respectively. Significant reconstruction of the surface occurs upon relaxation. The top-most $\mathrm{Mg}$ cations relax inward toward the next layer by $0.35 \AA$, which is about $35 \%$ of the distance between the $\mathrm{Mg}$ ions and the next O-Al-O layer. Half of the oxygen ions in the O-Al-O layer relax slightly away from their ideal position, toward the vacuum by $0.09 \AA$, while the other half relax in the opposite direction by $0.17 \AA$. The $\mathrm{Al}$ ions shift towards the bulk by $0.06 \AA$. A similar type of relaxation was found in the work of van der Laag et al. [18].

Using the minimized structures, we found that there are spurious effects that arise when we calculate dopant segregation profiles. In particular, within the bulk, while all Al sites are symmetrically equivalent, there are two different energies for dopant substitution, presumably the consequence of small surface dipoles related to the atomic structure of the surface. In an attempt to discover even more stable surface structures, we annealed the surfaces as detailed above. This led to simulation cells in which the dopant profiles were more symmetric and single valued in the bulk, which seemed more physical. These structures were used throughout the rest of the study.

Figure 2(d) shows the relaxed structure after annealing for the $\{100\} \mathrm{b}$ surface. Here, further relaxation and reconstruction at the surface compared to the minimized structure (Figure 2(c)) are visible. For example, one $\mathrm{Al}$ ion from the $\mathrm{O}-\mathrm{Al}-\mathrm{O}$ layer swaps position with a $\mathrm{Mg}$ layer in the subsurface, indicating that cation disordering processes are significantly easier at the surface than in the bulk and that the most stable surface structure may have some level of cation disorder, or inversion. Enhanced inversion at spinel surfaces was also reported by Rasmussen and co-workers [34]. Compared to the minimized surface (Figure 2(c)), in the annealed-plus-minimized surface, the O-Al-O layer relaxes slightly out $(\sim 0.05-0.07 \AA)$ while some of the top-most $\mathrm{Mg}$ ions relax in by an additional $\sim 0.40-0.60 \AA$ and take positions within the O-Al-O layer (as opposed to forming a distinct layer on the very top of the surface as in Figure 2(c)). Some of the original surface $\mathrm{Mg}$ ions even relax all the way to the subsurface $\mathrm{Mg}$ layer while others move from the subsurface to the top-most layer, indicating significant cation mobility within the surface.

The behavior illustrated in Figure 2 for the $\{100\} \mathrm{b}$ surface is generic to all of the surfaces we have examined. Figure 3 shows the final annealed-plus-minimized structures of the other surfaces. In the $\{100\}$ a surface, after annealing and within the top three surface cation layers, more than half of the $\mathrm{Mg}$ ions in the sub-surface layer swap positions with the nearest two $\mathrm{Al}$ ion layers, and some $\mathrm{Mg}$ ions were found at the surface after relaxation. The $\{110\} \mathrm{b}$ surface is very stable, structurally. The atomic structure of this surface essentially remained unchanged after annealing (as compared to the minimized-only structure). As compared to the as-constructed structure, all of the surface $\mathrm{Mg}$ ions moved into the unoccupied octahedral sites from their regular tetrahedral site positions but surface $\mathrm{Al}$ ions do not change their positions. The $\{110\}$ a surface exhibited significant reconstruction after annealing as compared to simply minimizing: one third of the surface $\mathrm{Al}$ ions moved into the sub-surface $\mathrm{Mg}$ ion position and those $\mathrm{Mg}$ from 
that layer relaxed to the top surface. Finally, considering the two $\{111\}$ surfaces, relaxation and reconstruction is more apparent in the $\{111\}$ a surface, both in the minimized and annealed-plusminimized structures as compared to the $\{111\}$ b surface. In the $\{111\}$ a surface, the four outermost cation layers are split into six layers upon relaxation and reconstruction whereas in the $\{111\}$ b surface this relaxation in not as strong. After annealing both of the $\{111\}$ surfaces show signs of inversion, with $\{111\} \mathrm{b}$ exhibiting inversion in the top two cation layers whereas $\{111\} \mathrm{a}$ shows inversion up to the fourth cation layer from the surface. In the $\{100\} b$ and $\{110\} b$, the surface layer is relatively dense whereas, in the other surfaces, there are some isolated islands where oxygen and $\mathrm{Al}$ cluster. This suggests that the reconstruction has not completed during the annealing stage, as the surfaces are very inhomogeneous in structure. However, further annealing led to no significant changes in the structure of the surface, indicating that, if the reconstruction is incomplete, there are large barriers inhibiting the formation of that reconstruction. That is, if there are lower energy reconstructions possible, the kinetics that dictate the evolution towards such structures are too slow to become active during our annealing simulations.

Comparing the structures of all of the surfaces considered, we find some general trends common for all surfaces: (i) cation inversion is prevalent at most of the annealed surfaces; (ii) compared to the as-constructed structure, the surface layer is disordered to some extent; and (iii) significant relaxation of oxygen occurs towards the surface. Overall, due to the complexity involved in this system, we have encountered diverse surface structures, which are further altered after annealing. It is worth noting that, under experimental conditions, low-index surfaces are expected to exhibit additional structural complexity, as oxide surfaces are strongly influenced not only by temperature but also by the oxygen environment (O-rich or O-poor). Moreover, the existence of defects such as dislocations [35], terraces, and ledges [36] on $\mathrm{MgAl}_{2} \mathrm{O}_{4}$ surfaces have been reported in experiments. This further complicates the atomic structure of $\mathrm{MgAl}_{2} \mathrm{O}_{4}$ surfaces. Although we have not addressed these additional defects in our work, the structural description reported here captures, at the most fundamental level, the basic trends in an extremely complex oxide surface, which can be used as a starting point to comprehend other type of defects and intricacies observed in literature. In what follows, we will focus on the interaction of dopants with these surfaces.

Table 1 summarizes the surface energies of the minimized and annealed-plus-minimized surfaces, highlighting the role that annealing has on further stabilizing the surface structure. Annealing stabilizes the surfaces by reducing the surface energy in almost all of the cases except for the $\{110\} \mathrm{b}$ surface, which maintains the same structure regardless of the annealing. In this case, we can only speculate that the ions are in very stable configurations and if more stable configurations are possible, they are not kinetically accessible during the course of our MD simulations. This is evident from the structure shown in Fig. 3, in which the surface ions in the $\{110\} \mathrm{b}$ surface are coordinated on both sides along the full periodicity of the model, a feature which is not observed in the other structures. It is also notable that after annealing most of the surfaces have very similar energies of $3.3-3.6 \mathrm{~J} / \mathrm{m}^{2}$. The exception is the most stable surface, the 
$\{100\} \mathrm{b}$ surface, which has a surface energy of $2.56 \mathrm{~J} / \mathrm{m}^{2}$. The lowest energy for each surface orientation is $2.56 \mathrm{~J} / \mathrm{m}^{2}$ for the $\{100\}$ surface, $3.27 \mathrm{~J} / \mathrm{m}^{2}$ for $\{110\}$ surface, and $3.45 \mathrm{~J} / \mathrm{m}^{2}$ for $\{111\}$ surface. The $b$-terminated surface is significantly more stable for the $\{100\}$ orientation, the a-terminated surface is slightly more stable for the $\{111\}$ surface, and the two terminations for the $\{110\}$ orientation are essentially degenerate in energy. Thus, only the energy of the $\{100\}$ surface is very sensitive to the chemical termination of the surface. Overall, these energies lie between various experimental estimates [37,38]. In particular, that the $\{100\}$ surface is the most stable agrees with experimental measurements that found the $\{100\}$ surface has the lowest fracture surface energy [38].

\section{Segregation of Dopants}

Utilizing the annealed-plus-minimized surface structures, dopant energy profiles were calculated as discussed in the Methods section. Figure 4(a) shows energy profiles for $\mathrm{La}^{3+}$ at all of the six surface structures, revealing the energetic dependence of substituting $\mathrm{La}$ as a function of distance from each surface. The energy is measured relative to the lowest energy position for the dopant within a given surface structure. In all cases, the site with the lowest energy cost for placing a dopant is either at the surface layer or at the subsurface layer. As the dopant position is moved from either of the two surfaces in each simulation cell towards the bulk, the energy quickly increases and reaches a constant value in the bulk.

There are a few interesting points to be made from these profiles. First, the interaction of La with the surface is typically short ranged, though there is variation depending on the surface. For the $\{111\}$ a surface, the interaction is greatest, with the dopant-surface interaction extending by about $15 \AA$ from the surface. In contrast, for the $\{110\} \mathrm{b}$ surface, the interaction is less than $10 \AA$. Further, not all sites near the surface are favorable for the dopant. In particular, at the $\{111\} \mathrm{a}$ surface, some sites are almost $1.0 \mathrm{eV}$ higher in energy than in the bulk, suggesting that the dopant structure at this interface may be rather complicated. This occurs at other surfaces as well, but to a much lesser extent. Finally, the preferred site is not always at the top-most surface layer of $\mathrm{Al}$ ions, but rather in the layers underneath, again highlighting the complicated nature of dopant segregation to these surfaces.

Figure 4(b) shows the energy profiles for all four dopants within the $\{100\} \mathrm{b}$ surface structure, the most stable of the six surfaces we have examined. The La dopant has the largest segregation energy of the dopants considered. For trivalent dopants, the segregation energy increases with the size of the dopant ion $\left(r_{L a^{3+}}=1.045 \AA, r_{G d^{3+}}=0.938 \AA\right.$, and $\left.r_{Y^{3+}}=0.900 \AA[39,40]\right)$. Dopants with larger ionic radius have higher mismatch with the host cation $\left(r_{A l^{3+}}=0.530 \AA\right.$ [39]) and thus create more elastic strain within the lattice. They thus have greater segregation energy to the surface, where the extra freedom of the surface atomic structure can accommodate these ions. The tetravalent dopant $\mathrm{Zr}\left(r_{Z r^{4+}}=0.720 \AA\right.$ [39]) does not follow the same trend because it has a valence different than the host atom and both strain and electrostatic effects will play a role in its behavior. Zr has slightly higher segregation energy than $\mathrm{Y}$ even though the ionic 
radius is significantly smaller. This suggests that differences in valence are more effective than differences in radius mismatch in dictating the segregation of impurities. We note that we have not considered the role that charge-compensating defects would have in influencing the segregation of aliovalent dopants. In the case of aliovalent dopant segregation to grain boundaries in $\mathrm{CeO}_{2}$, we found that, due to electrostatic interactions between defects and dopants, charge compensating defects strongly segregate to the grain boundaries along with the dopants [41]. However, considering the role of these defects, which bring in additional complexity, is beyond the scope of the present study, particularly since the defect compensation mechanism for a supervalent dopant is likely rather complex, as the defect chemistry in non-stoichiometric spinel is also complex [42-44].

Similar trends for segregation to the six surface structures were found for all of the dopants at the six interfaces considered in this study. The calculated segregation energies are summarized in Figure 5. For each surface, La has the highest segregation energy. For a given surface orientation, all of the dopants prefer the same termination. For the $\{100\}$ and $\{111\}$ surfaces, the a-termination exhibits higher segregation energies compared to the b-terminated surface whereas, for the $\{110\}$ surface, the b-termination has higher segregation energy than the aterminated surface. However, interestingly, as the nature of the dopant changes, the hierarchy of which surfaces more strongly attract the dopant changes. All dopants segregate most strongly to the $\{111\}$ a surface, but while the segregation of La is weakest to the $\{100\} \mathrm{b}$ surface, the $\{110\} \mathrm{a}$ surface is the weakest for the other dopants. There is also a crossover in which dopants prefer the $\{111\} \mathrm{b}$ and the $\{110\} \mathrm{b}$ surface, with $\mathrm{Zr}$ segregating more strongly to the $\{111\} \mathrm{b}$ surface and the trivalent dopants segregating more strongly to the $\{110\} \mathrm{b}$ surface, although the differences are small. This indicates that, while overall trends are not sensitive to the details of either the nature of the dopant or the structure of the surface, the details certainly are.

Finally, we note that there is little correlation between surface energy and dopant segregation behavior. The two $\{110\}$ surfaces have essentially degenerate surface energies but the $\{110\} \mathrm{b}$ surface consistently attracts dopants more strongly. Also, segregation is strongest to the $\{111\}$ a surface, but this surface does not have the highest surface energy. Clearly, other factors such as local chemical environment of surface segregation sites dictate dopant segregation to these surfaces and the surface energy alone is not a predictor of these trends.

\section{Surface Energy of Doped Surfaces}

In Figure 6, we convert the segregation energies depicted in Figure 5 to surface energies, via Eq 2. The overall effect of the doping is the noticeable drop in surface energies. As the dopant is changed from $\mathrm{Zr}$ to $\mathrm{Y}$ to $\mathrm{Gd}$ to $\mathrm{La}$, the surface energy is consistently lowered, suggesting that selective doping can stabilize spinel surfaces. The trends reported in Figure 6 indicate that for applications at the nanoscale, where surface behavior in spinel oxides will play a critical role, careful doping can be utilized as an effective strategy to stabilize the nanostructure. This is not surprising, as the benefits of doping have been reported in previous work. For instance, recent 
experiments have reported that manganese dissolution at spinel surfaces can be suppressed via doping [45]. Even in cases where the surface crystallinity of nanoparticles may be low, doping has been shown to reduce surface energy, though these same experiments show that particles on the size of $10 \mathrm{~nm}$ can still exhibit relatively high crystallinity [11,12]. We also note that, for undoped spinel, the $\{111\}$ a surface is the second least stable surface, but for La-doped spinel, it is nearly the second most favored surface, suggesting that by adding certain types of dopants, the relative stability of individual facets can be altered. That is, the energetics of these surfaces are not uniformly reduced via doping, but some surfaces are affected more strongly than others. As discussed earlier, this could be particularly useful in scenarios where enhancing the fraction of specific facets could lead to improvement in overall properties [13-15].

\section{Predicted Equilibrium Morphology}

Finally, using the lowest energy termination for each of the $\{100\},\{110\}$, and $\{111\}$ surfaces, the equilibrium crystallite morphology was predicted using the Wulff method. The predicted equilibrium crystal shapes are shown in Figure 7. Without annealing, all of the faces are present but the shape of the crystal has essentially a cubic morphology with a predominance of $\{100\}$ faces, a few $\{110\}$ edges, and very minimal $\{111\}$ facets on the corners of the cube. Annealing changes the relative stability of the different surfaces and thus the shape: more $\{111\}$ and fewer $\{110\}$ faces are present using the annealed-plus-minimized surfaces than when using the minimized-only surfaces. Doping further stabilizes the $\{111\}$ surfaces relative to the $\{110\}$ surfaces and consequently further modifies the morphology, with an even larger fraction of $\{111\}$ surface area. As discussed, these results highlight the potential to use doping to alter crystal morphology and thus influence surface-sensitive properties, such as catalytic behavior.

\section{DISCUSSION}

The surface energies we obtained for the undoped relaxed spinel surfaces are slightly higher than that found in the work of Davies et. al. and Fang et al. [16, 17], although the relative stability of the surfaces are the same. The difference in the absolute value is because of the potential parameters. Both of those prior works used potential parameters derived by Lewis and Catlow [46], while we used potential paramaters derived by Busker et al. [28], a choice made because of the avilability of potential paramaters for various dopant species that are consistent with those for the Al- and Mg-oxides.

The segregation profiles for all dopants to all surfaces are qualitatively similar and every surface and dopant combination results in positive segregation energies, indicating that all of the dopants will segregate to each of the surfaces, though some of the dopant-surface interactions do exhibit complex behavior in which some of the sites at the surface are effectively repulsive. At the $\{111\}$ a surface, the most favorable position for the dopant was not precisely at the surface layer, but rather beneath the surface. Similar behavior was observed by Stanek et al.[47] for $\mathrm{Zr}^{4+}$ segregation in $\mathrm{UO}_{2}\{111\}$ surface. 
The dependency of the segregation energy on ionic radius and charge of the dopant is consistent at each of the surfaces. However, we find that ionic valence has a somewhat stronger influence on the dopant segregation as compared to ionic size. Hong et al. [48] reported the segregation energies of several fission products to $\mathrm{UO}_{2}$ grain boundary and attributed the prepensity of segregation to ionic charge, ionic radius, and the particular segregation site. Analogous to the trend reported here, they found that fission products with similar ionic charge as the host uranium ion exhibit weaker segregation, while those with dissimilar ionic charge segregate more strongly.

Regarding the predicted crystal morphology, there is some experimental evidence supporting our result that the $\{100\}$ surface is the lowest energy surface of spinel. Investigating the fracture behavior of single crystal of spinel in different orientations, Stewart et al. [38] found that the fracture surface energy and elastic modulus are a minimum for $\{100\}$ orientation at room temperature. Recently, Massaro et al. [21] systematically studied and identified the most stable structure for $\{100\}$ and $\{110\}$ facets in $\mathrm{MgAl}_{2} \mathrm{O}_{4}$ spinel. Their study, in which they performed a detailed configurational analysis of these two facets, relied primarily on empirical potentials, but results for the $\{100\}$ facets were validated with hybrid DFT. In agreement with our work, they found the $\mathrm{Mg}$ termination to be the most stable $\{100\}$ surface and the Al-O termination to be the most stable $\{110\}$ surface (though we find this termination to be essentially degenerate with the $\mathrm{Mg}$ termination and they find a more significant difference in energetics between the two terminations). While our results qualitatively agree with the empirical calculation results of Massaro et al., there are quantitative differences. This discrepancy primarily stems from the usage of different force field parameters. However, other factors could be the different system sizes employed (our models are larger than those employed by Massaro et al.) or our use of 3D slab models and their use of reduced periodicity 2D models, which has been shown in the past to give somewhat different results from one another [49]. More importantly, however, for the $\{100\}$ surface, the physical trends they find using empirical potentials are confirmed with DFT, providing confidence in the trends we report here as well. Overall, the basic trends pertaining to the relative stability of low-index $\mathrm{MgAl}_{2} \mathrm{O}_{4}$ spinel facets reported in this study are consistent with other empirical and DFT trends in literature, and thus provides confidence to utilize these models to further study the effect of dopant at various facets, which is the main goal of our work.

The surface energies of all of the low index surfaces were lowered by doping with each of the four different dopants considered. This work gives insight into how to stabilize the surfaces of spinels, and complex oxides more generally, through surface energy reduction by choosing an appropriate dopant. This can aid the nanomaterials community to stabilize nanoparticles through a thermodynamic approach. Moreover, we also observed that the relative stability of different surfaces can be changed using dopants which can inform catalyst materials design which require specific surface orientations. A key result in our work is that, by changing the relative surface energy of each crystal face, dopants can change the surface morphology of spinel crystals. Similar conclusions have been reached for perovskites [33]. 


\section{CONCLUSION}

Atomistic modeling was carried out on three low-index spinel surfaces to determine their structure, stability, and the behavior of dopants near the surfaces. High-temperature annealing stablizes the surfaces, relative to direct energy minimization, through reconstructions that are activated thermally. The $\{100\} \mathrm{b}$ surface is the lowest energy surface, in agreement with previous calculations. Further, it is significantly more stable than the next lowest energy surface. After annealing, the energetics of the other surfaces are all very similar.

By systematically doping each system from the bulk to the surface, the relative segregation tendencies of three trivalent dopants and one tetravalent dopant were determined. Isovalent dopants with larger ionic radii tend to segregate more strongly to the surface as they have greater size mismatch with the host $\mathrm{Al}$ ion. The ionic valence mismatch between the dopant and the host ion also leads to stronger segregation. Among the dopants considered, La exhibits the strongest segregation to each type of surface. The segregation energy also varies with the type of surface but does not follow any simple relationship. In particular, there is no correlation between segregation and surface energetics. Thus, the segregation energy depends on both the nature of the dopant and the detailed atomic structure of the surface. The results and trends reported here suggest that selective doping could be exploited to stabilize the complex surfaces of spinel oxides leading to enhancement in their properties for diverse nanoscale applications.

Equivalently, doping not only reduces the surface energy of all of the surfaces, but it also changes the relative stability of some surfaces. This leads to changes in the predicted equilibrium morphology of spinel crystals. A greater amount of $\{111\}$ surfaces are present in the doped spinel crystals than in the undoped spinel crystals. This suggests that doping can be one route for improving the catalytic properties of spinels.

\section{ACKNOWLEDGEMENTS}

R.C. and M.H. thank the U.S. Department of Energy - Early Career Program Award BES ER46795 for support. P.P.D. acknowledges support by the UC Lab Fees Research Program 12LF- 239032. B.P.U. acknowledges support by the U.S. Department of Energy, Office of Science, Basic Energy Sciences, Materials Sciences and Engineering Division. Los Alamos National Laboratory is operated by Los Alamos National Security, LLC, for the National Nuclear Security Administration of the (U.S.) Department of Energy under contract DE-AC52-06NA25396. M. M.H. acknowledges the computational resources provided by Professor Roland Faller at UC Davis. 


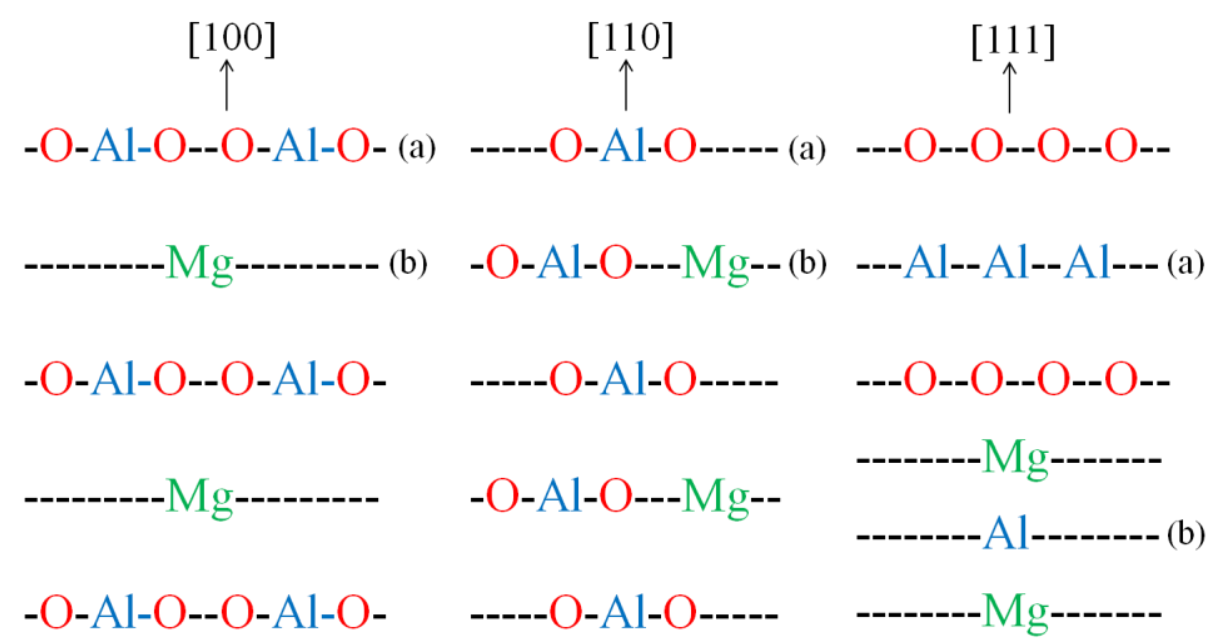

Fig. 1 Schematic stacking sequence of the three low index surface orientations of spinel 


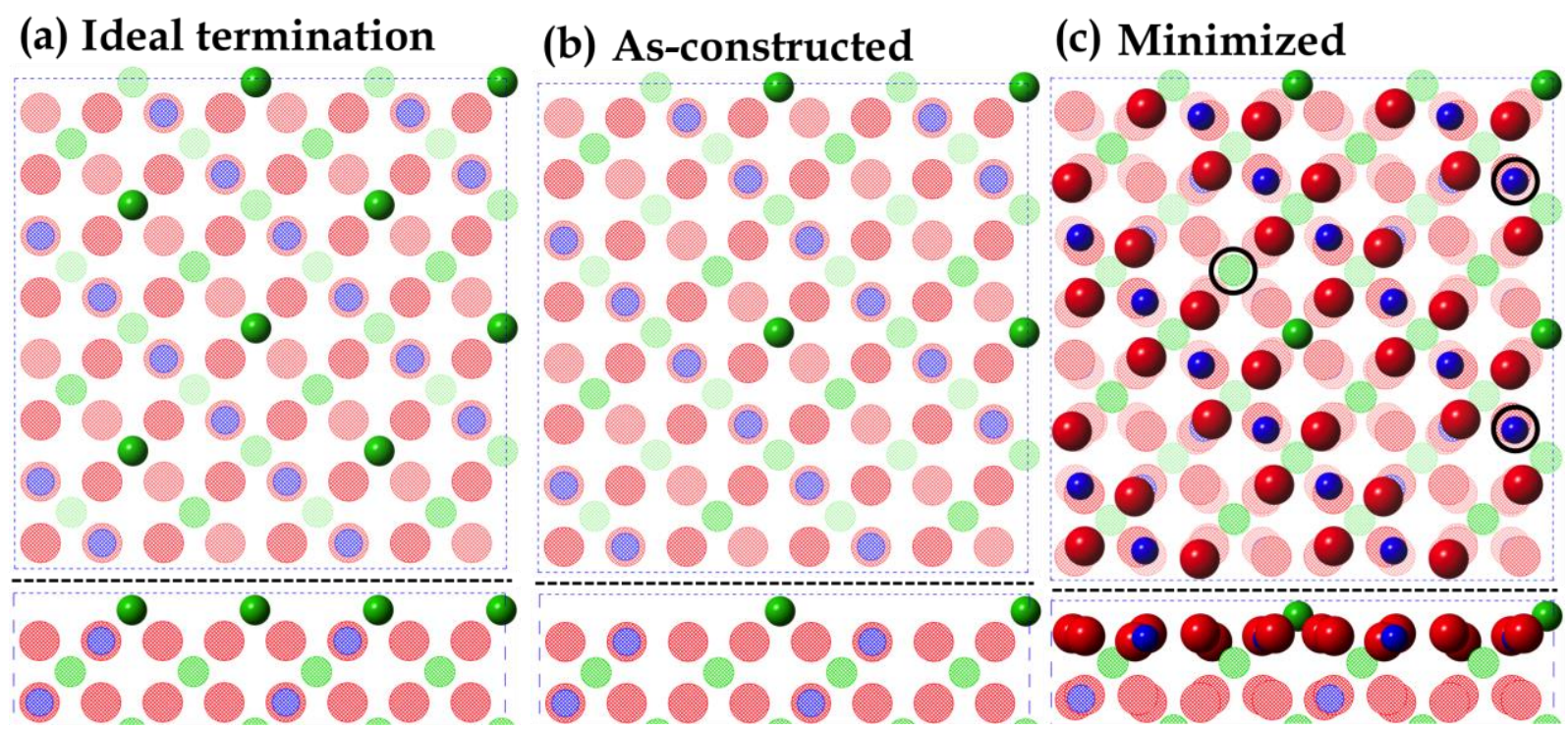

(d) Annealed + Minimized

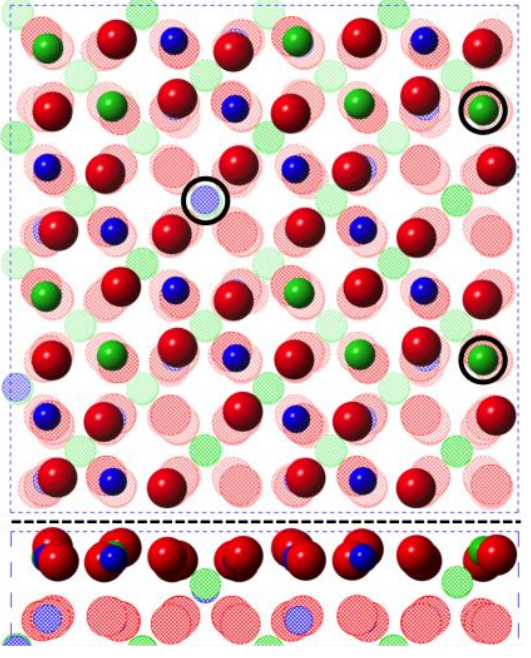

Fig. 2 Structure of the $\mathrm{MgAl}_{2} \mathrm{O}_{4}\{100\} \mathrm{b}$ surface (the lowest energy surface in our calculations). In each frame, the surface is shown from a perspective normal to the surface (top panel) as well as from the side (bottom panel). The black dashed line separates the two panels in each part of the figure. (a) Ideal surface as cleaved at the Mg layer, (b) $\{100\} \mathrm{b}$ termination after half of the ions have been moved to the opposing surface, (c) relaxed surface found after directly minimizing the structure in (b), (d) annealed-plus-minimized surface found after first annealing the structure in (b) with high temperature $\mathrm{MD}$ and then minimizing. $\mathrm{Mg}, \mathrm{Al}$, and $\mathrm{O}$ are green, blue, and red respectively. The surface layer ions, highlighted with the solid rendered spheres, were identified by a distance criterion wherein the surface layer atoms are the topmost, and atoms that are at a distance greater than $0.7 \AA$ in the direction normal to that surface layer are considered as subsurface layer atoms. The subsurface layer ions are indicated by the cross- 
hatched spheres, whose relative depth is indicated by the brightness of the corresponding sphere. Solid black circles indicate a selection of locations where cation inversion has occurred.

(a) $\{100\} a$

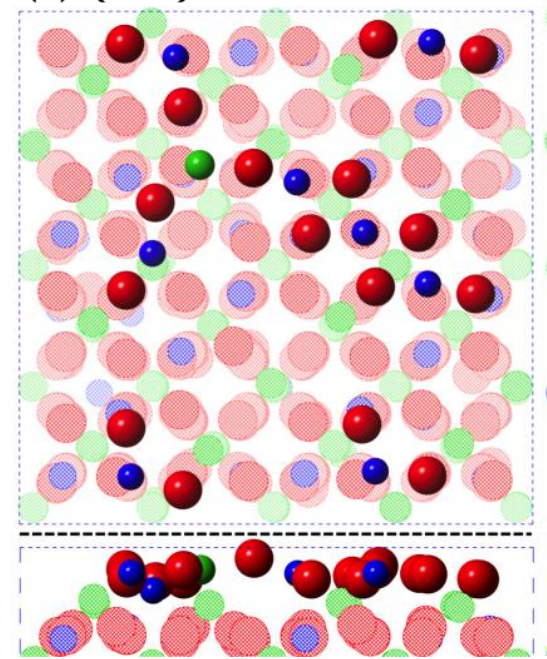

(d) $\{110\} b$

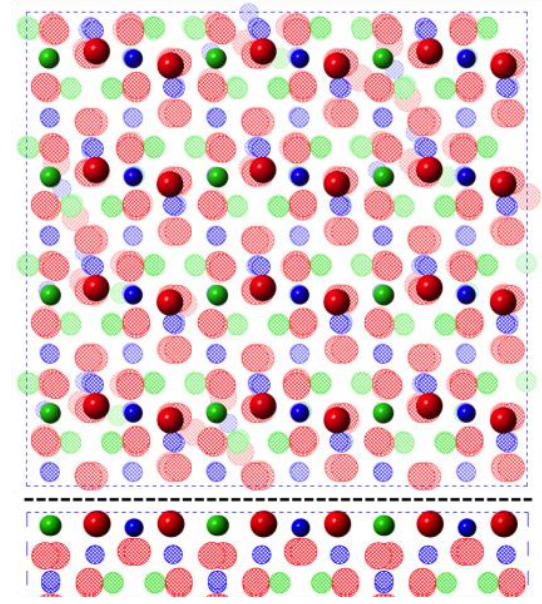

(b) $\{100\} b$

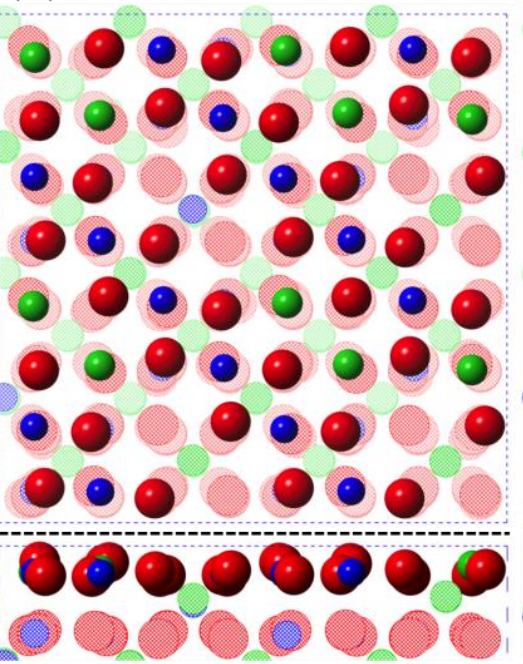

(e) $\{111\} a$

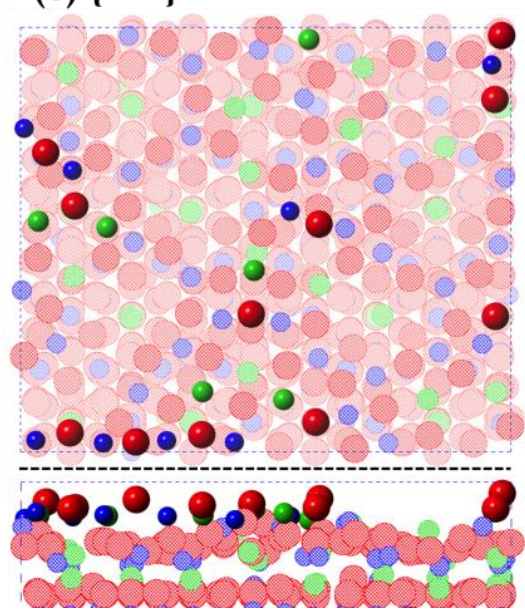

(c) $\{110\} a$

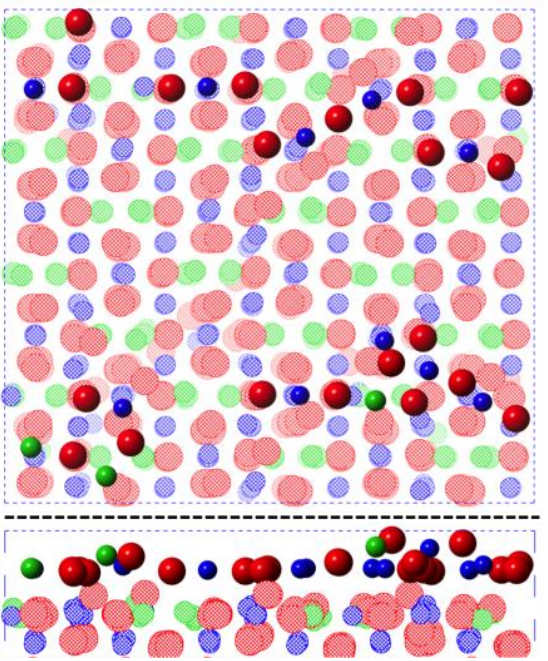

(f) $\{111\} b$

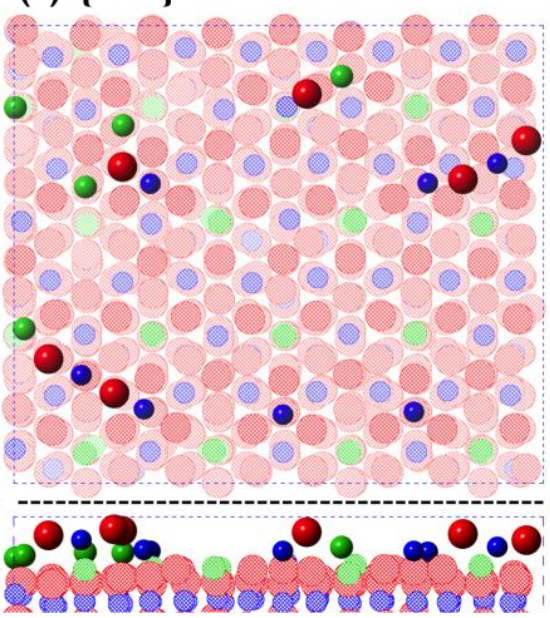

Fig. 3 Relaxed structures after annealing of all three surfaces, each with two different terminations. The color scheme and structure of the panels is the same as in Figure 2. Figure $3 \mathrm{~b}$ is the same as Figure $2 \mathrm{~d}$ and is included here for ease of comparison and completeness. 

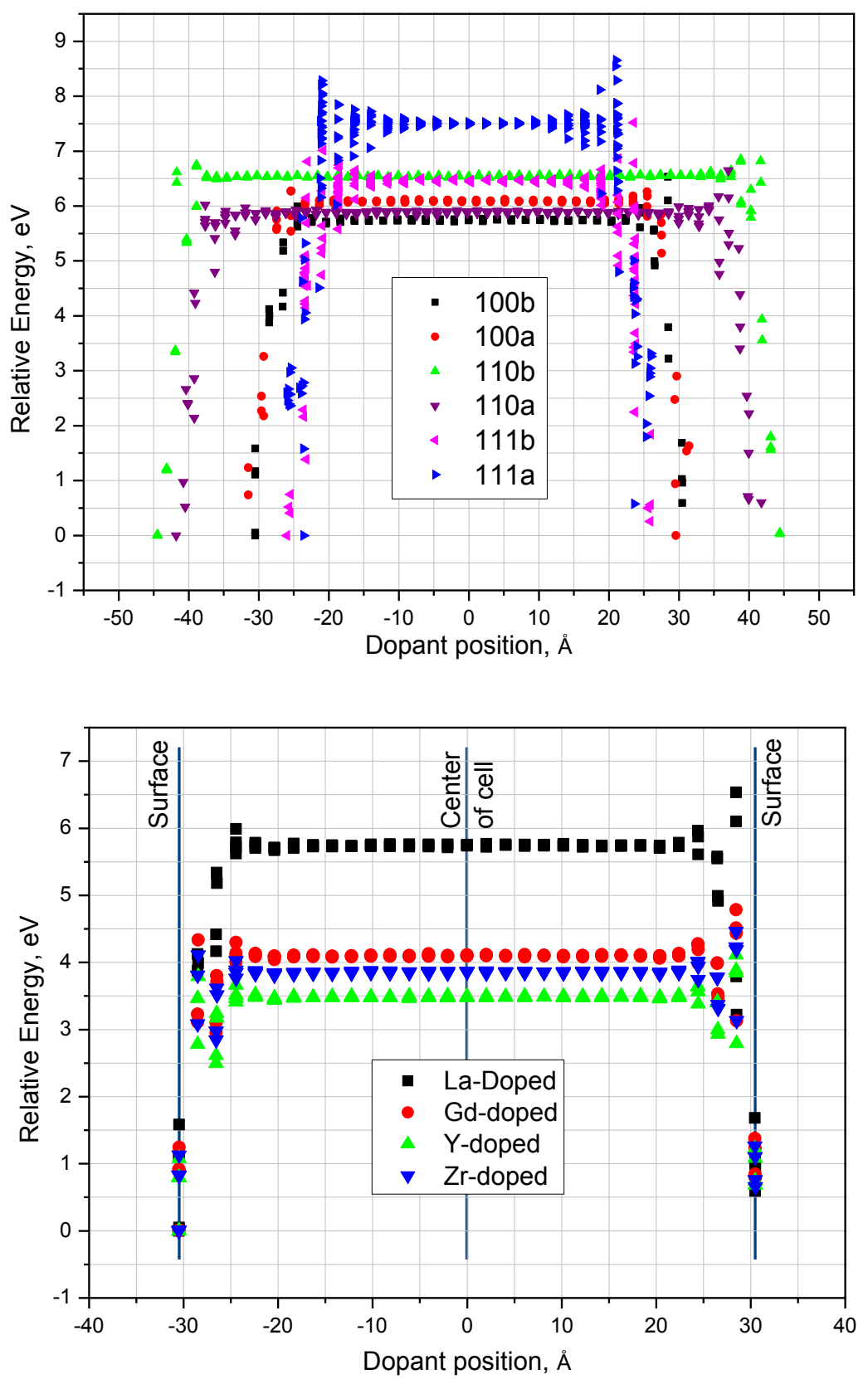

Fig. 4 (a) Energy profiles of $\mathrm{La}^{3+}$ as a function of distance from the center of the simulation cell as it is substituted for $\mathrm{Al}^{3+}$ near the six surface structures considered. For each surface, the energy of the lowest energy site for La substitution is taken as the zero of energy. Note that the two surfaces in each simulation cell are not identical after the annealing procedure described in the text and so the profiles are not completely symmetric. (b) Energy profiles for the four dopants considered near the $\{100\} \mathrm{b}$ surface, the most stable surface as identified by the atomistic simulations, as a function of dopant position. The lines in (b) identify the position of the center of the simulation cell as well as the positions of the two surfaces for clarity. 


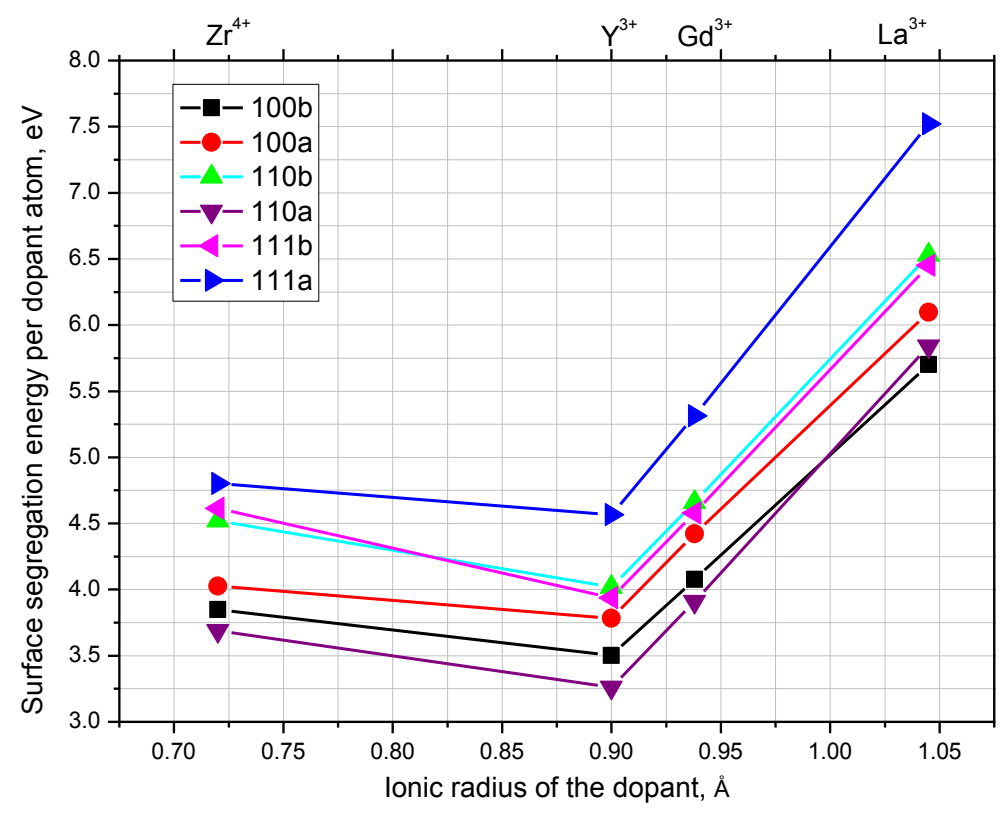

Fig. 5 Segregation energies (of a single dopant atom) of the four different dopants considered to all six low-index surfaces considered in this work.

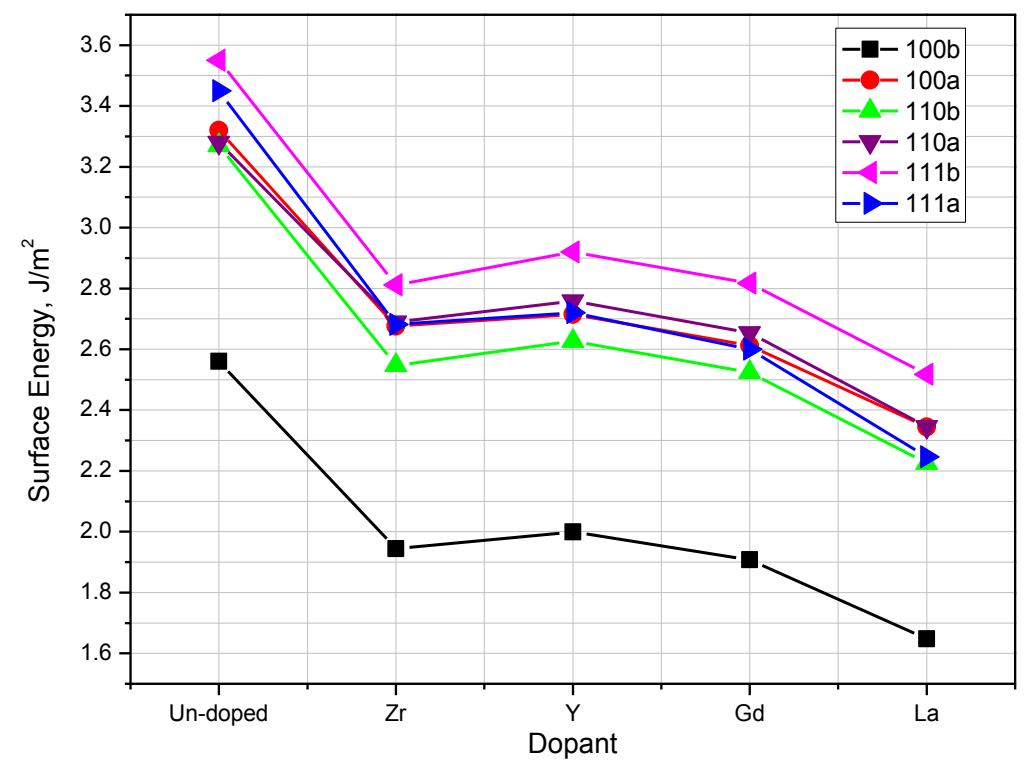

Fig. 6 Surface energies of six undoped (annealed-plus-minimized) and doped spinel surfaces for the four different dopants considered as determined from Eq. 2 and the segregation energies presented in Figure 4. 

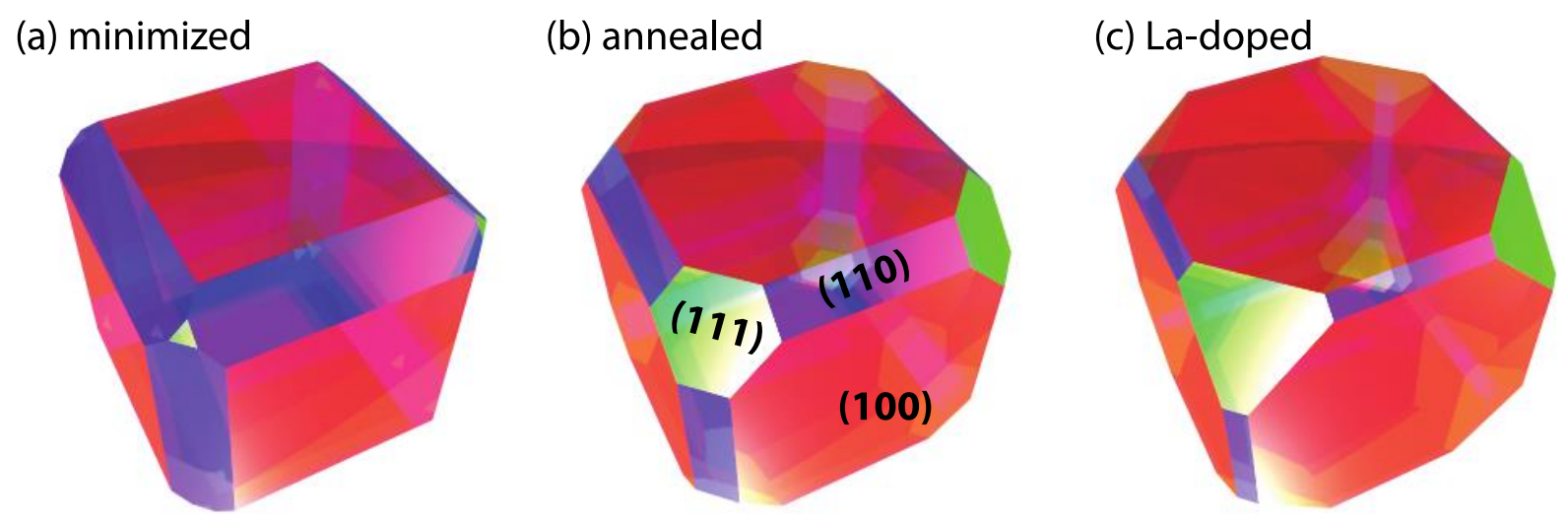

Fig. 7 Predicted equilibrium morphologies for spinel crystals using surfaces that were (a) minimized, (b) annealed-plus-minimized, and (c) doped with La.

Table $1 \quad$ Surface Energies of undoped spinel

\begin{tabular}{ccc}
\hline Surface & $\begin{array}{c}\text { Minimized only, } \\
\mathbf{J} / \mathbf{m}^{\mathbf{2}}\end{array}$ & $\begin{array}{c}\text { Annealed-plus-minimized, } \\
\mathbf{J} / \mathbf{m}^{\mathbf{2}}\end{array}$ \\
\hline$\{100\} \mathrm{b}$ & 2.70 & 2.56 \\
$\{100\} \mathrm{a}$ & 3.90 & 3.32 \\
$\{110\} \mathrm{b}$ & 3.27 & 3.27 \\
$\{110\} \mathrm{a}$ & 4.36 & 3.28 \\
$\{111\} \mathrm{a}$ & 4.16 & 3.45 \\
$\{111\} \mathrm{b}$ & 3.92 & 3.55 \\
\hline
\end{tabular}




\section{REFERENCES}

1. Campbell, C.T., Ultrathin metal films and particles on oxide surfaces: structural, electronic and chemisorptive properties. Surface science reports, 1997. 27(1): p. 1-111.

2. Renaud, G., Oxide surfaces and metal/oxide interfaces studied by grazing incidence $X$-ray scattering. Surface Science Reports, 1998. 32(1): p. 5-90.

3. Zheng, H., et al., Multiferroic BaTiO3-CoFe2O4 nanostructures. Science, 2004. 303(5658): p. 661663.

4. Odette, G., M. Alinger, and B. Wirth, Recent developments in irradiation-resistant steels. Annu. Rev. Mater. Res., 2008. 38: p. 471-503.

5. Choudhury, S., D. Morgan, and B.P. Uberuaga, Massive interfacial reconstruction at misfit dislocations in metal/oxide interfaces. Scientific reports, 2014. 4.

6. Kingery, W., H. Bowen, and D. Uhlmann, Introduction to ceramics, 1976. Jhon Willey \& Sons, New York.

7. Gibbs, J.W., On the equilibrium of heterogeneous substances. American Journal of Science, 1878(96): p. 441-458.

8. Kirchheim, R., Reducing grain boundary, dislocation line and vacancy formation energies by solute segregation. I. Theoretical background. Acta Materialia, 2007. 55(15): p. 5129-5138.

9. Kirchheim, R., Reducing grain boundary, dislocation line and vacancy formation energies by solute segregation: II. Experimental evidence and consequences. Acta Materialia, 2007. 55(15): p. 5139-5148.

10. Weissmüller, J., Alloy effects in nanostructures. Nanostructured Materials, 1993. 3(1): p. 261272.

11. Wu, L., et al., Surface Segregation on Manganese Doped Ceria Nanoparticles and Relationship with Nanostability. The Journal of Physical Chemistry C, 2014. 118(51): p. 30187-30196.

12. Chang, C.-H., et al., Thermodynamic Stability of SnO2 Nanoparticles: The Role of Interface Energies and Dopants. The Journal of Physical Chemistry C, 2015. 119(11): p. 6389-6397.

13. Rennard, R. and J. Freel, The role of sulfur in deactivation of PtMgAl2O4 for propane dehydrogenation. Journal of Catalysis, 1986. 98(2): p. 235-244.

14. Bocanegra, S., et al., Effect of the Synthesis Method of MgAl2O4 and of Sn and Pb Addition to Platinum Catalysts on the Behavior in n-Butane Dehydrogenation. Industrial \& Engineering Chemistry Research, 2010. 49(9): p. 4044-4054.

15. Li, W.-Z., et al., Stable platinum nanoparticles on specific MgAl2O4 spinel facets at high temperatures in oxidizing atmospheres. Nature communications, 2013. 4.

16. Davies, M.J., S.C. Parker, and G.W. Watson, Atomistic simulation of the surface structure of spinel. J. Mater. Chem., 1994. 4(6): p. 813-816.

17. Fang, C.M., S.C. Parker, and G. With, Atomistic simulation of the surface energy of spinel MgAl2O4. Journal of the American Ceramic Society, 2000. 83(8): p. 2082-2084.

18. Van der Laag, N., et al., Geometry of $\{001\}$ Surfaces of Spinel (MgA/204): First-Principles Simulations and Experimental Measurements. Journal of the American Ceramic Society, 2005. 88(6): p. 1544-1548.

19. Lennie, A., et al., Structures of Fe 304 (111) surfaces observed by scanning tunneling microscopy. Physical Review B, 1996. 53(15): p. 10244.

20. Zasada, F., et al., Periodic DFT and HR-STEM studies of surface structure and morphology of cobalt spinel nanocrystals. Retrieving 3D shapes from 2D images. The Journal of Physical Chemistry C, 2011. 115(14): p. 6423-6432. 
21. Massaro, F.R., M. Bruno, and F. Nestola, Configurational and energy study of the (100) and (110) surfaces of the MgAl 204 spinel by means of quantum mechanical and empirical techniques. CrystEngComm, 2014. 16(39): p. 9224-9235.

22. Perkins, J., G. West, and M. Lewis, Analysis and spectroscopy of rare earth doped magnesium aluminate spinel. Advances in applied ceramics, 2005. 104(3): p. 131-134.

23. West, G., J. Perkins, and M. Lewis, Characterisation of fine-grained oxide ceramics. Journal of materials science, 2004. 39(22): p. 6687-6704.

24. Wulff, G., On the question of speed of growth and dissolution of crystal surfaces. Z. Kristallogr, 1901. 34: p. 449-530.

25. Plimpton, S., Fast parallel algorithms for short-range molecular dynamics. Journal of computational physics, 1995. 117(1): p. 1-19.

26. Buckingham, R.A. The classical equation of state of gaseous helium, neon and argon. in Proceedings of the Royal Society of London A: Mathematical, Physical and Engineering Sciences. 1938. The Royal Society.

27. Ewald, P., Evaluation of optical and electrostatic lattice potentials. Ann. Phys, 1921. 64: p. 253287.

28. Busker, G., et al., Solution mechanisms for dopant oxides in yttria. Journal of the American Ceramic Society, 1999. 82(6): p. 1553-1559.

29. Tasker, P., The stability of ionic crystal surfaces. Journal of Physics C: Solid State Physics, 1979. 12(22): p. 4977.

30. Bertaut, F., The electrostatic term of the surface energy. CR Acad Sci, 1958. 246: p. 3447.

31. Tasker, P., The surface energies, surface tensions and surface structure of the alkali halide crystals. Philosophical Magazine A, 1979. 39(2): p. 119-136.

32. Abramowski, M., Atomistic simulations of the uranium/oxygen system. PhD Thesis, University of London, UK, 2001.

33. Alfredsson, M., et al., Dopant control over the crystal morphology of ceramic materials. Surface Science, 2007. 601(21): p. 4793-4800.

34. Rasmussen, M.K., et al., Stable cation inversion at the MgAl 204 (100) surface. Physical review letters, 2011. 107(3): p. 036102.

35. Yanina, S.V. and C.B. Carter, Dislocations at spinel surfaces. Surface science, 2002. 511(1): p. 133-146.

36. Yanina, S.V. and C.B. Carter, Terraces and ledges on (001) spinel surfaces. Surface science, 2002. 513(2): p. L402-L412.

37. Navrotsky, A., et al., Nanophase transition metal oxides show large thermodynamically driven shifts in oxidation-reduction equilibria. science, 2010. 330(6001): p. 199-201.

38. Stewart, R. and R. Bradt, Fracture of single crystal MgA/2O4. Journal of Materials Science, 1980. 15(1): p. 67-72.

39. Shannon, R.t. and C.T. Prewitt, Effective ionic radii in oxides and fluorides. Acta Crystallographica Section B: Structural Crystallography and Crystal Chemistry, 1969. 25(5): p. 925-946.

40. Shannon, R. and C. Prewitt, Revised values of effective ionic radii. Acta Crystallographica Section B: Structural Crystallography and Crystal Chemistry, 1970. 26(7): p. 1046-1048.

41. Dholabhai, P.P., et al., Structure and segregation of dopant-defect complexes at grain boundaries in nanocrystalline doped ceria. Physical Chemistry Chemical Physics, 2015.

42. Andersson, D.A. and C.R. Stanek, Mixing and non-stoichiometry in Fe-Ni-Cr-Zn-O spinel compounds: density functional theory calculations. Physical Chemistry Chemical Physics, 2013. 15(37): p. 15550-15564.

43. Murphy, S., et al., Non-stoichiometry in MgAl2O4 spinel. Philosophical Magazine, 2010. 90(10): p. 1297-1305. 
44. Ball, J., et al., Defect processes in MgAl 204 spinel. Solid State Sciences, 2008. 10(6): p. 717-724.

45. Lu, J., et al., Effectively suppressing dissolution of manganese from spinel lithium manganate via a nanoscale surface-doping approach. Nature communications, 2014. 5.

46. Lewis, G. and C. Catlow, Potential models for ionic oxides. Journal of Physics C: Solid State Physics, 1985. 18(6): p. 1149.

47. Stanek, C., M. Bradford, and R. Grimes, Segregation of Ba2t, Sr2t, Ce4t and Zr4t to UO2 surfaces. Journal of Physics: Condensed Matter, 2004. 16(27): p. S2699.

48. Hong, M., et al., The role of charge and ionic radius on fission product segregation to a model UO2 grain boundary. Journal of Applied Physics, 2013. 113(13): p. 134902.

49. Evarestov, R. and A. Bandura, Hartree-Fock calculations of electronic structure of (110)-surface of rutile TiO2: Comparison of single (2D) and periodic (3D) slab models. International journal of quantum chemistry, 2004. 96(3): p. 282-291. 